Research Article

\title{
Botanical Drug Puerarin Ameliorates Liposaccharide-Induced Depressive Behaviors in Mice via Inhibiting RagA/mTOR/p70S6K Pathways
}

\author{
Jia Zhao $\mathbb{D}^{1,2,3,4}$ Yizhen Jia, ${ }^{1}$ Wei Zhao $\mathbb{D}^{2}{ }^{2}$ Huixin Chen, ${ }^{2}$ Xiuying Zhang, ${ }^{2}$ Fung Yin Ngo, ${ }^{2}$ \\ Dan Luo, ${ }^{2}$ Youqiang Song $\left(\mathbb{D},{ }^{5}\right.$ Lixing Lao $\mathbb{1},{ }^{2}$ and Jianhui Rong $\mathbb{D}^{2,6}$ \\ ${ }^{1}$ Department of Chinese Medicine, The University of Hong Kong Shenzhen Hospital, Shenzhen, China \\ ${ }^{2}$ School of Chinese Medicine, The University of Hong Kong, 10 Sassoon Road, Pokfulam, Hong Kong, China \\ ${ }^{3}$ Zhu Nansun's Workstation, School of Chinese Medicine, The University of Hong Kong, 10 Sassoon Road, Pokfulam, \\ Hong Kong, China \\ ${ }^{4} Y u$ Jin's Workstation, School of Chinese Medicine, The University of Hong Kong, 10 Sassoon Road, Pokfulam, Hong Kong, China \\ ${ }^{5}$ School of Biomedical Science, The University of Hong Kong, 21 Sassoon Road, Pokfulam, Hong Kong, China \\ ${ }^{6}$ The University of Hong Kong Shenzhen Institute of Research and Innovation (HKU-SIRI), Shenzhen, China \\ Correspondence should be addressed to Jianhui Rong; jrong@hkucc.hku.hk
}

Received 10 May 2021; Revised 27 September 2021; Accepted 29 September 2021; Published 18 October 2021

Academic Editor: Xiaolei Shi

Copyright ( 2021 Jia Zhao et al. This is an open access article distributed under the Creative Commons Attribution License, which permits unrestricted use, distribution, and reproduction in any medium, provided the original work is properly cited.

\begin{abstract}
Background. The depressive symptom hallmarks the progression of the neurodegenerative diseases, especially Alzheimer's disease. Bacterial infection is related to inflammation and depression. The present project thereby examined whether botanical drug puerarin could attenuate liposaccharide- (LPS-) induced depressive behaviors in mice. Methods. Adult male C57BL/6N mice were sequentially treated with LPS and puerarin and evaluated for the depressive behaviors by tail suspension test and forced swim test. The brain tissues were profiled for the molecular targets of puerarin by next-generation RNA sequencing technique. Candidate targets were further verified in LPS-treated mice, neural stem cells, and highly differentiated PC12 cell line. Results. Puerarin ameliorated LPS-induced depression in the mice. RNA sequencing profiles revealed that puerarin altered the expression of 16 genes while markedly downregulated Ras-related GTP-binding protein A (RagA) in LPS-treated mice. The effect of puerarin on RagA expression was confirmed by immunostaining, Western blot, and quantitative real-time PCR (qRTPCR). Biochemical studies showed that puerarin inhibited RagA/mTOR/p70S6K pathway, attenuated the accumulation of mTORC1 in close proximity to lysosome, and reduced the production of proinflammatory cytokines. Conclusions. Botanical drug puerarin attenuated inflammation and depressive behaviors in LPS-challenged mice by inhibiting RagA/mTOR/p70S6K pathways. Puerarin may be a lead compound for the new antidepressant drugs.
\end{abstract}

\section{Introduction}

Alzheimer's disease (AD) is characterized by memory loss and cognitive deficit due to progressive neurodegeneration [1]. Most of AD patients suffer from depression while severe depression affects over 264 million people (https://www.who .int/). Infection and social stress dramatically increase the prevalence of depression worldwide [2]. The etiology of the depression remains elusive. Among several risk factors, neuroinflammation is recently identified to be highly related to depression [3]. The proinflammatory cytokines including interleukin- (IL-) $1 \beta$ and IL- 6 were increased whereas the anti-inflammatory cytokines including IL-10 and IL-4 were decreased in depression [4]. Moreover, bacterial lipopolysaccharide (LPS) induces inflammation and depression in mice [5-7]. Our previous study showed that LPS induced 
depression in mice by upregulating small guanosine triphosphatases (GTPases) RagA expression and subsequently activating mammalian target of rapamycin (mTOR)/p70S6K pathway [8]. Therefore, the RagA/mTOR/p70S6K pathway may be a therapeutic target for the treatment of depression.

The mTOR pathway is a key mechanism in the regulation of protein synthesis, cellular metabolism, and autophagy while mTOR is also implicated in the proliferation of neural stem cells and circadian rhythms [9]. Two different mTOR complexes (e.g., mTORC1 and mTORC2) execute the biological functions in response to specific stimuli [10]. The complex mTORC1 facilitates the cell growth by inhibiting autophagy and the expression of lysosomal genes whereas mTORC2 phosphorylates and activates PKB/Akt and PKC kinase, thereby promoting cell survival [11]. The mTORC1 pathway is dysregulated in the neurodegenerative diseases [12]. Nutrients promote mTORC1 translocation to the lysosomal membrane and activate the mTORC1 pathway via Rag GTPases [13]. On the other hand, Rag GTPases exist in four isoforms, namely, RagA, RagB, RagC, and RagD, and often form heterodimers (e.g., RagA/B, RagC/D). Upon stimulation by the nutrients, the complex RagA/B binds guanosine triphosphate (GTP) whereas the complex RagC/D binds guanosine diphosphate (GDP). The activation of Rag GTPases promotes the lysosomal localization of mTORC1 to facilitate the interaction of mTORC1 with the kinase activator Rheb $[14,15]$. The active mTORC1 colocalizes with the lysosomal biomarker lysosome-associated membrane protein (LAMP2) and regulates the lysosomal biogenesis [16, 17]. Moreover, the activation of mTORC1 induces the phosphorylation of the downstream targets including p70 S6 kinase (p70S6K) and eukaryotic translation initiation factor 4E-binding protein 1 (4E-BP1), thereby driving the onset of depression $[18,19]$.

The conventional antidepressant drugs have been found to have various side effects in the past couple of decades [20]. As an example, ketamine exhibits rapid antidepressant effects via regulating 4E-BPs [19]. However, ketamine causes various side effects and even toxicity because its abuse harms people and society [21]. On the other hand, natural products could effectively attenuate LPS-induced depression in mice [22]. Along this line, our group recently demonstrated that C-glucosylated isoflavone puerarin (Figure 1(a)), as the main active compound from the herbal medicine Radix puerariae, attenuated pain and depression in mice with spared nerve injury [23]. Consistently, other studies also showed that puerarin attenuated depressive-like behaviors by upregulating $\mathrm{FGF}_{2}$ expression or via estrogen and BDNF signaling pathways $[24,25]$. Thus, it would be of importance to further dissect the mechanism by which puerarin exhibits antidepressant activity.

In the present study, we hypothesized that puerarin could attenuate the depressive-like behaviors in LPSchallenged mice. Following puerarin treatment, depressivelike behaviors were assessed in the LPS-challenged mice. The brain tissues were subjected to the transcriptome profiling for the molecular targets of puerarin. The candidate targets of puerarin were further verified in LPS-treated mice, neural stem cells, and highly differentiated PC12 cell line.

\section{Materials and Methods}

2.1. Antibodies and Biochemical Reagents. The antibodies against RagA, phospho-mTOR, mTOR, phospho-p70S6K, p70S6K, and GAPDH were obtained from Cell Signaling Technology (Boston, MA, USA). HRP-conjugated antirabbit IgG was purchased from Sigma-Aldrich (St. Louis, MO, USA). NeuN and LAMP2 antibodies were purchased from Abcam (Cambridge, MA, USA). Alexa Fluor 488conjugated goat anti-mouse IgG and Alexa Fluor 594conjugated goat anti-rabbit IgG were obtained from Invitrogen (Carlsbad, CA, USA). ChamQ SYBR qPCR master mix was obtained from Vazyme (Nanjing, China). QuantiNova SYBR green PCR kit was obtained from QIAGEN (Valencia, CA, USA). Dulbecco's modified Eagle's medium (DMEM), DMEM/F12, fetal bovine serum (FBS), horse serum (HS), N2, B27, penicillin, streptomycin, basic fibroblast growth factor (bFGF), and epidermal growth factor (EGF) were obtained from Thermo Fisher Scientific (Waltham, MA, USA). The mammalian expression vector pcDNA3.1 was obtained from Invitrogen (Carlsbad, CA, USA). Puerarin was obtained from Yick-Vic Chemicals \& Pharmaceuticals (Hong Kong, China). LPS, Accumax solution, RIPA buffer, and other reagents were obtained from Sigma-Aldrich (St. Louis, MO, USA) unless otherwise indicated.

2.2. Animals and Drug Administration. Animal experiments were approved by the Committee on the Use of Live Animals in Teaching and Research of the University of Hong Kong (CULATR 4284-17). Male C57BL/6N mice (7-8 weeks, $18-25 \mathrm{~g}$ ) were provided by the Centre for Comparative Medicine Research, the University of Hong Kong. Animals were housed on a $12 \mathrm{~h}$ light-dark cycle and allowed to freely access food and water at the animal facility, the University of Hong Kong. For the behavioral experiments, puerarin was dissolved in saline containing 50\% 1,2-propylene glycol. LPS was dissolved in the saline. Mice were randomly separated into 5 groups $(n=5)$ : control, LPS, LPS+puerarin $(30 \mathrm{mg} / \mathrm{kg})$, LPS+puerarin $(60 \mathrm{mg} / \mathrm{kg})$, and LPS+puerarin $(120 \mathrm{mg} / \mathrm{kg})$. As outlined in Figure 1(b), following intragastric administration of puerarin for $24 \mathrm{~h}$, mice sequentially received oral gavage of puerarin and intraperitoneal injection of LPS $(0.083 \mathrm{mg} / \mathrm{kg})$ with $1 \mathrm{~h}$ interval for another $24 \mathrm{~h}$, and the mice in the control group were given the same volume of saline with or without 1,2-propylene glycol. The behavioral tests were performed after LPS treatment.

2.3. Behavioral Tests. Following drug treatment, mice were assessed for depressive behaviors by tail suspension test (TST) and forced swim test (FST) as previously described $[23,26]$.

For TST, adult mice were immobilized to the suspension bar by attaching the tail with adhesive tape. The distance of 20-25 cm was kept between apparatus floor and mouse nose. During the test over $6 \mathrm{~min}$, a blinded observer recorded the immobility time of the mice.

For FST, adult mice were transferred into a plastic cylin$\operatorname{der}\left(30 \mathrm{~cm}\right.$ high, $20 \mathrm{~cm}$ diameter) filling water at $22 \pm 2^{\circ} \mathrm{C}$ to 


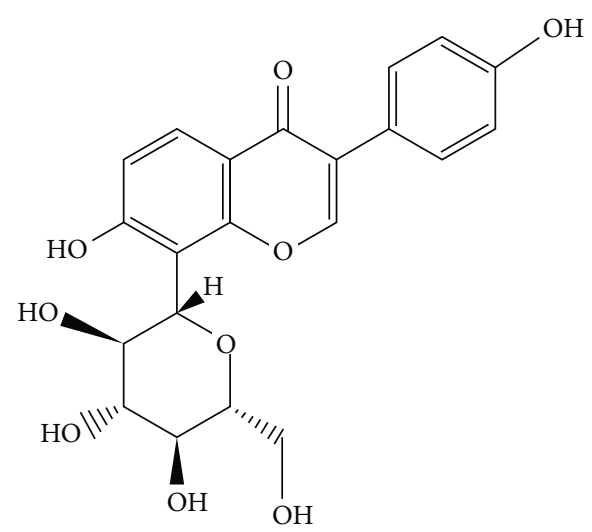

(a)

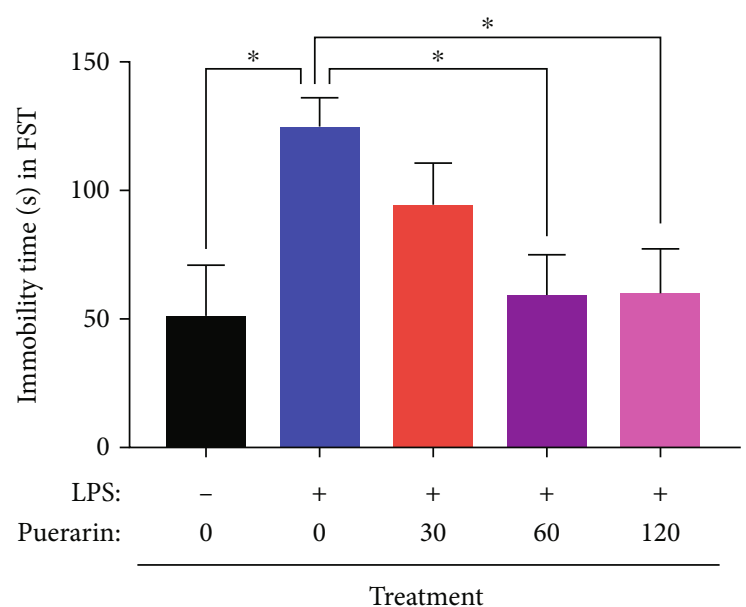

(c)

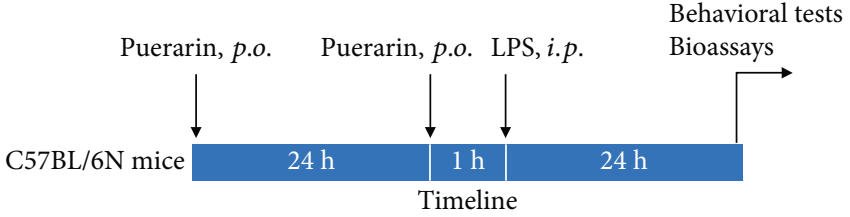

(b)

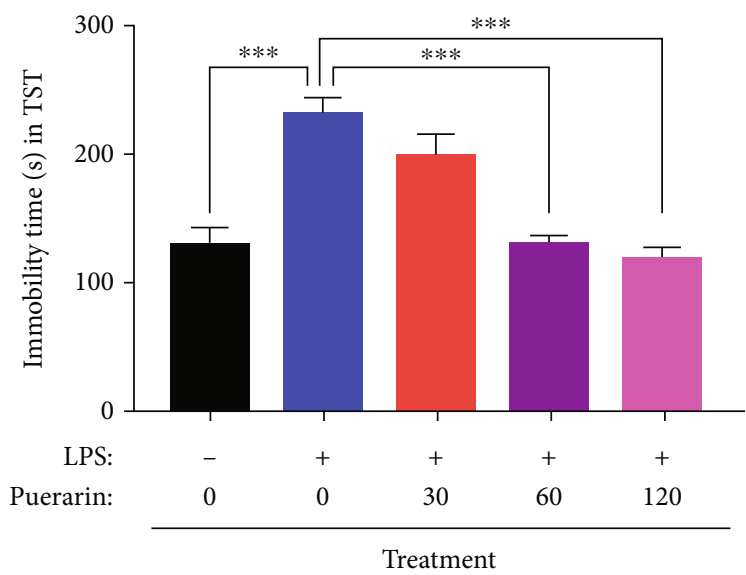

(d)

FIGURE 1: Puerarin ameliorated depressive-like behaviors in the LPS-challenged mice. (a) The chemical structure of puerarin was shown. (b) The design of the animal experiments. Mice were separated into 5 groups: control, LPS, and LPS+puerarin (30, 60, or 120 mg/kg). After drug treatment, mice were assessed for depressive-like behaviours, and brain tissue was collected for the bioassays. (c) Forced swim test (FST). The depressive-like behaviors of mice were evaluated by measuring immobility time during 4 min. (d) Tail suspension test (TST). The depressive-like behaviors of mice were evaluated by measuring immobility time during $6 \mathrm{~min}$. The data were presented as means \pm SEM $(n=6) .{ }^{*} p<0.05 ;{ }^{* *} p<0.01 ;{ }^{* * *} p<0.001$.

the height of $15 \mathrm{~cm}$. Following 2 min habituation sessions, the immobility time was recorded by a blinded observer during the last $4 \mathrm{~min}$.

2.4. Transcriptome Profiling by Next-Generation RNA Sequencing. Following the behavioral tests, the $3 \mathrm{~mm}$ frontal cortex was collected from the mouse brains $(n=3)$. The RNAs were extracted with TRIzol reagent from Thermo Fisher Scientific (Waltham, MA, USA) and purified with RNeasy plus mini kit from QIAGEN (Valencia, CA, USA). Following reverse transcription, the cDNA libraries were sequenced on the Illumina HiSeq 4000 System in BGI (Shenzhen, China). The bioinformatic analyses were performed essentially as previously described [27, 28]. Briefly, the sequence reads were mapped to the mouse grcm38_ snp_tran reference sequence with HTSAT2 [29]. Ballgown was applied for the differential gene expressions [30]. The differential genes were selected based on $p$ value of $<0.05$ and fold change (FC) of $\geq 2$. $R$ was used to make the heatmap. The network of the differential genes was established by Cytoscape (https://cytoscape.org/). The differential genes were analyzed with the Kyoto Encyclopedia of Genes and Genomes (KEGG) pathway and the enriched Gene Ontology (GO) by DAVID 6.8 (http://david.ncifcrf.gov). The $p$ values less than 0.05 indicated that the differential genes were significantly enriched [31].

2.5. Molecular Cloning. RagA cDNA was prepared by polymerase chain reaction (PCR) amplification by using the forward primer (5'-GTACAAGCTTCAGGTGATGCCCAA TACA- $\left.3^{\prime}\right)$ and the reverse primer $\left(5^{\prime}\right.$-GTACGATATCG GCATTATTTCAACGCATGA-3 ${ }^{\prime}$ ). PCR amplification was conducted in $50 \mu \mathrm{l}$ reaction mixture containing $10 \mu \mathrm{l}$ of $5 \mathrm{X}$ HF buffer, $2 \mu \mathrm{l}$ of $50 \mathrm{mM} \mathrm{MgCl} 2,1 \mu \mathrm{l}$ of $25 \mathrm{mM}$ dNTPs mix, $2.5 \mu \mathrm{l}$ each of $20 \mu \mathrm{M}$ reverse and forward primers, $100 \mathrm{ng}$ of template DNA, and $0.5 \mu \mathrm{l}$ of Phusion DNA polymerase $(5 \mathrm{U} / \mu \mathrm{l}, \mathrm{NEB}, \mathrm{USA})$ in a programmable Eppendorf Thermocycler (Hong Kong, China). PCR reaction was programmed as follows: an initial denaturation of 30 seconds 
at $98^{\circ} \mathrm{C}, 35$ cycles of $98^{\circ} \mathrm{C}$ for 10 seconds, $57^{\circ} \mathrm{C}$ for 30 seconds, and $72^{\circ} \mathrm{C}$ for $1 \mathrm{~min}$, with a final extension at $72^{\circ} \mathrm{C}$ for $10 \mathrm{~min}$. PCR product was purified from gel by the gel extraction kit (QIAGEN, USA). After the cleavage with HindIII and EcoRV, RagA PCR product was purified and ligated into mammalian expression vector pcDNA 3.1(+) from Invitrogen (Carlsbad, CA, USA). Following transformation and selection, pcDNA 3.1-RagA plasmid DNA was prepared in DH5 $\alpha$ E. coli cells and purified with QIAGEN Plasmid Miniprep kit (Valencia, CA, USA). The clones were sequenced through Sanger DNA Sequencing Facility at BGI, Shenzhen, China.

2.6. Neural Stem Cell (NSC) Culture. Cortical NSCs were produced from $\mathrm{C} 57 \mathrm{BL} / 6 \mathrm{~N}$ mouse embryos on embryonic day 14 as previously described $[32,33]$. The NSCs were plated in a $90 \mathrm{~mm}$ dish at the density of $1 \times 10^{5}$ cells $/ \mathrm{ml}$ and cultured in DMEM/F12 medium supplemented with $2 \% \mathrm{~B} 27,1 \% \mathrm{~N} 2,20 \mathrm{ng} / \mathrm{ml} \mathrm{bFGF}, 20 \mathrm{ng} / \mathrm{ml}$ EGF, and $1 \%$ streptomycin and penicillin at $37^{\circ} \mathrm{C}$ for 5-7 days. The single cell suspension of NSCs was seeded in the 6-well plates coated with poly-D-lysine for $24 \mathrm{~h}$ and treated with $200 \mathrm{ng} / \mathrm{ml}$ LPS with or without puerarin at the doses of 10 , 25 , and $50 \mu \mathrm{M}$ for $24 \mathrm{~h}$.

\subsection{Highly Differentiated PC12 Cell Culture and} Transfection. Highly differentiated PC12 cell line was obtained from National Collection of Authenticated Cell Cultures (Shanghai, China). The cells were cultured in DMEM supplemented with $10 \%$ HS, 5\% FBS, $1 \%$ penicillin and streptomycin at $37^{\circ} \mathrm{C}$, and $5 \% \mathrm{CO}_{2}$. For the transfection, the cells were seeded in the 6-well plates at the density of $1.5 \times 10^{5}$ cells $/ \mathrm{ml}$ for $24 \mathrm{~h}$. The pcDNA3.1(+) vector or pcDNA3.1(+)-RagA plasmid was transfected into the cells by using Effectene Transfection Reagent from QIAGEN (Valencia, CA, USA) for $24 \mathrm{~h}$.

2.8. Western Blot Analysis. The frontal cortex tissues or primary mouse neural stem cells were lysed in RIPA buffer for $30 \mathrm{~min}$. The cellular proteins $(30 \mu \mathrm{g})$ or brain tissue proteins $(70 \mu \mathrm{g})$ were separated by $10 \%$ SDS-polyacrylamide gels. The PVDF membranes were blocked in the 5\% BSA in Trisbuffered saline and $0.1 \%$ Tween-20 buffer overnight at $4^{\circ} \mathrm{C}$. Blots were incubated with primary antibodies overnight and secondary antibodies for $3 \mathrm{~h}$. Subsequently, blots were detected with Amersham ${ }^{\mathrm{TM}}$ ECL $^{\mathrm{TM}}$ Select Western blotting detection reagents from GE Healthcare (Uppsala, Sweden) [34].

2.9. Immunostaining. After the behavioral tests, a mixture solution of ketamine/xylazine was used for the mice anesthesia, and $4 \%$ paraformaldehyde (PFA) in $0.01 \mathrm{M}$ phosphatebuffered saline (PBS) was used for the perfusion. 4\% PFA was used to fix the mouse brains overnight at $4^{\circ} \mathrm{C}$. The thickness of the coronal sections of the $3 \mathrm{~mm}$ frontal cortex was $40 \mu \mathrm{m}$. NeuN and RagA primary antibodies were used to probe the tissues overnight at $4^{\circ} \mathrm{C}$. The tissues were then incubated with secondary antibodies including Alexa Fluor 488-conjugated goat anti-mouse IgG and Alexa Fluor 594conjugated goat anti-rabbit IgG for $2 \mathrm{~h}$ at room temperature (RT). 4'-6-Diamidino-2-phenylindole (DAPI) was used to stain the cell nucleus. The slides were examined under a Zeiss LSM 700 confocal microscopy (Jena, Germany). The images were analysed by NIH ImageJ software (http:// imagej.net/ImageJ2) [23].

For cell culture, the NSCs were treated with $200 \mathrm{ng} / \mathrm{ml}$ LPS with or without puerarin at the doses of 10,25, and $50 \mu \mathrm{M}$ for $24 \mathrm{~h}$ and fixed with $4 \%$ PFA. Blocking was performed in $5 \%$ normal goat serum for $2 \mathrm{~h}$ at RT. The NSCs were sequentially incubated with primary antibodies against mTOR and LAMP2 (FITC) overnight at $4^{\circ} \mathrm{C}$ and Alexa Fluor 594 goat anti-rabbit IgG secondary antibody for $2 \mathrm{~h}$ at RT. DAPI was used to stain the cell nucleus. The slides were examined under a Carl Zeiss fluorescence microscopy (Jena, Germany) [8].

2.10. Quantitative Real-Time PCR ( $q R T-P C R)$. The mouse frontal cortex tissues and highly differentiated PC12 cells were analysed for gene expression by qRT-PCR technique as previously described [35]. Briefly, total RNAs were extracted from the frontal cortex tissues and highly differentiated PC12 cells with TRIzol reagent from Thermo Fisher Scientific (Waltham, MA, USA). The cDNAs were prepared from total RNAs with RevertAid RT Reverse Transcription kit from Thermo Fisher Scientific (Waltham, MA, USA). PCR detection was performed with a SYBR Green PCR kit from QIAGEN (Valencia, CA, USA) and Vazyme (Nanjing, China). The primers for various mouse genes were obtained from QIAGEN as follows: RagA (Mm_Rraga_1_SG; QT00257880), FZD1 (Mm_Fzd1_1_SG; QT00290542), Fitm2 (Mm_Fitm2_1_SG; QT00147903), Camkk2 (Mm_Camkk2_ 1_SG; QT00162449), Ap3S2 (Mm_Ap3s2_1_SG; QT00148428), Mesdc1(Mm_Mesdc1_1_SG; QT00292187), GAPDH (Mm_Gapdh_3_SG; QT01658692), and $\beta$-actin (Mm_Actb_1_SG; QT 00095242). The primers for rat genes were as follows: RagA (NM_053973), F: 5 '-GGAACCTGG TGCTGAACCTGTG-3'， R: 5'-GGATGGCTTCCAGACA CGACTG-3' ; IL-6 (NM_012589), F: 5'-GCCCACCAGGA ACGAAAGTCAA-3 ${ }^{\prime}, \quad$ R: $5^{\prime}$-GGAAGGCAGTGGCTGT CAACAA- $3^{\prime}$; IL-1 $\beta$ (NM_031512), F: 5' $5^{\prime}$-AATGCCTCGTG CTGTCTGACC- $3^{\prime}$, R: $5^{\prime}$-GTGGGTGTGCCGTCTTTCA TCA- ${ }^{\prime}$; $\beta$-actin (NM_031144), F: $5^{\prime}$-GTATGCCTCTGGTC GTACCA-3', R: 5-CTCTCAGCTGTGGTGGTGAA-3'. The data were normalized to $\beta$-actin or GAPDH. The relative expression level of genes was indicated by $2-\Delta \Delta \mathrm{Ct}$.

2.11. Statistical Analysis. The data were presented as mean \pm SEM for body weight, behavioral experiments, and qRTPCR in the animal experiments and mean \pm SD for other experiments. The comparisons of multiple groups were performed using one-way analysis of variance (ANOVA) followed by Dunnett's test. GraphPad Prism 7 (La Jolla, CA, USA) was used to analyze the data. The $p$ values of less than 0.05 were regarded as statistically significant.

\section{Results}

3.1. Puerarin Attenuated the Depressive-like Behaviors in LPS-Challenged Mice. FST and TST were used to assess the 
effect of puerarin on the depressive behaviors in LPSchallenged mice as previously described [23]. As outlined in Figure 1(b), C57BL/6N mice were administered with LPS or in combination with puerarin. The results of FST and TST were shown in Figures 1(c) and 1(d), respectively. Indeed, compared with the control group, LPS markedly prolonged the immobility time of mice in the FST and TST. Puerarin $(60$ and $120 \mathrm{mg} / \mathrm{kg})$ significantly reduced the immobility time in LPS-treated mice in FST and TST compared with LPS alone group while such drug $(30 \mathrm{mg} / \mathrm{kg})$ did not exhibit evident activities.

3.2. Puerarin Affected RagA and Other 15 Genes in LPSTreated Mice. To discover the antidepressant mechanisms for puerarin, the mouse frontal cortex tissues were collected from control, LPS, and LPS+P groups and profiled by nextgeneration RNA sequencing technology. As shown in Figures 2(a) and 2(b), a total of 16 differentiated genes with $p<0.05$ and $\mathrm{FC} \geq 2$ were identified based on the comparison groups: LPS vs. control and LPS+P vs. LPS. In particular, 10 genes were upregulated while 6 genes were downregulated in the comparison of LPS $+\mathrm{P}$ group vs. LPS group. Puerarin downregulated the level of RagA expression to the largest extent $(\mathrm{FC}=0.02)$ based on RagA expression in LPS $+\mathrm{P}$ group and LPS group. The interaction network was created by including 16 differentiated genes and 7 closely related genes although no significant change was detected. The selected genes were enriched by GO and KEGG pathways. As shown in Figure 2(c), activate transcription factor (GO:0051091), Wnt-activated receptor (GO:0042813), and frizzled binding (GO:0005109) were the most significantly enriched in the GO terms "biological process" and "molecular function." Wnt-signaling pathway was the most significantly enriched KEGG pathway (KEGG: mmu04310).

3.3. Puerarin Effectively Downregulated RagA in LPSChallenged Mice. To verify the results of RNA sequencing, six representative genes (i.e., RagA, FZD1, Fitm2, Camkk2, Ap3S2, and Mesdc1) were validated by qPCR technique. As shown in Figure 3(a), the brain tissues from LPS group resulted in higher mRNA levels of RagA, FZD1, and Fitm2. Puerarin $(60 \mathrm{mg} / \mathrm{kg})$ significantly decreased the mRNA levels of these genes against LPS stimulation. RagA was further verified by Western blotting and immunostaining. As shown in Figures 3(b) and 3(c), LPS significantly enhanced the protein levels of RagA whereas puerarin $(60 \mathrm{mg} / \mathrm{kg})$ significantly reduced RagA expression in LPS-treated mice. Puerarin group showed the lower level of RagA expression compared with control group. As shown in Figures 3(d) and 3(e), LPS significantly enhanced the immunofluorescence staining of RagA expression whereas puerarin $(60 \mathrm{mg} / \mathrm{kg})$ significantly decreased the immunofluorescence intensity for RagA staining against LPS stimulation. Moreover, after LPS treatment, RagA and NeuN appeared to be colocalized in the frontal cortex of mice.

3.4. Puerarin Inhibited the Activation of RagA/mTOR/p70S6K Pathway and the Lysosomal Translocation of mTORC1 in Neural Stem Cells. To determine the mechanisms by which
RagA mediates the activity of puerarin, we focused on RagAassociated mTOR signaling pathway. For the effects of puerarin on the $\mathrm{RagA} / \mathrm{mTOR} / \mathrm{p} 70 \mathrm{~S} 6 \mathrm{~K}$ pathway, as shown in Figures 4(a) and 4(b), LPS treatment enhanced the protein expression of RagA, phospho-mTOR and phospho-p70S6K in neural stem cells. Puerarin $(50 \mu \mathrm{M})$ significantly decreased RagA expression against LPS stimulation. Moreover, puerarin $(25$ and $50 \mu \mathrm{M})$ significantly decreased the expression of phospho-mTOR and phospho-p70S6K against LPS stimulation. For the effects of puerarin on the lysosomal translocation of mTORC1, as shown in Figures 4(c) and 4(d), LPS enhanced the expression of lysosomal biomarker LAMP2 and promoted the colocalization of MTOR and LAMP2 in the nucleus. Puerarin reduced LPS-induced LAMP2 expression and disturbed the colocalization of MTOR and LAMP2 in a concentrationdependent manner. Puerarin $(50 \mu \mathrm{M})$ spread the distribution of LAMP2 in the cytosol of neural stem cells against LPS stimulation.

3.5. Puerarin Inhibited LPS-Induced Expression of Proinflammatory Cytokines. To study the role of RagA in the anti-inflammatory activity of puerarin, the effects of puerarin were evaluated on LPS-induced inflammatory responses in mice. Firstly, we clarified whether LPSinduced sickness in mice. After $24 \mathrm{~h}$ treatment, we measured the body weight of mice. As shown in Figure 5(a), LPS and puerarin did not obviously change the body weight of mice, suggesting that LPS did not cause sickness in mice. Secondly, we determined whether puerarin could suppress LPSinduced production of proinflammatory cytokines in mice. As shown in Figure 5(b), qRT-PCR analysis validated that LPS markedly induced the mRNA levels of proinflammatory genes including IL- 6 , IL- $1 \beta$, and TNF- $\alpha$. Puerarin effectively reduced the mRNA levels of IL- 6 and IL- $1 \beta$ and affected TNF- $\alpha$ to a lesser extent. Thirdly, we examined the effect of ectopic RagA overexpression on cytokine synthesis. The full-length RagA cDNA was cloned in pcDNA3.1 (+) vector and subsequently introduced into highly differentiated PC12 cells. As shown in Figure 5(c), RagA transfection resulted profound increase in the expression of RagA mRNA. The mRNA level of IL- 6 but not IL- $1 \beta$ was obviously elevated in RagA-transfected PC12 cells compared with that in pcDNA3.1 vector transfected cells.

\section{Discussion}

Depressive symptom may be one of the predictive signs for the onset of $\mathrm{AD}$ in the elderly population. Inflammation is known to increase the risk of depression in $\mathrm{AD}$ patients [36]. Thus, LPS was used to induce acute inflammation and depressive-like behaviors in mice [37]. Indeed, we recently found that LPS could cause the depressive-like behaviors in mice via activating RagA/mTOR/p70S6K pathway [8]. As for the therapy of depression, the conventional antidepressant drugs are challenged by gastrointestinal and sexual side effects $[38,39]$. Interestingly, we recently discovered that botanical drug puerarin could effectively attenuate depression and pain in mice with SNI [23]. Based on the side-by-side comparison with the clinical drugs, puerarin 


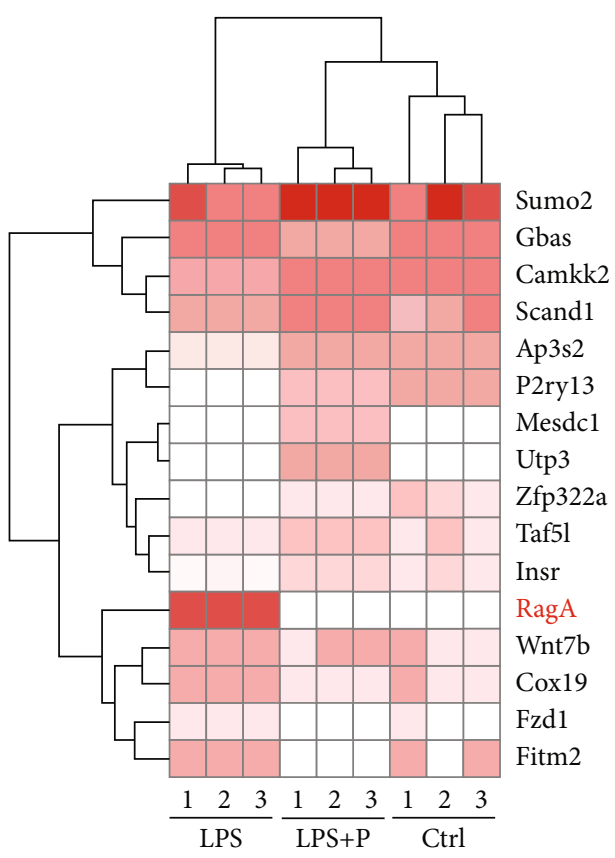

(a)

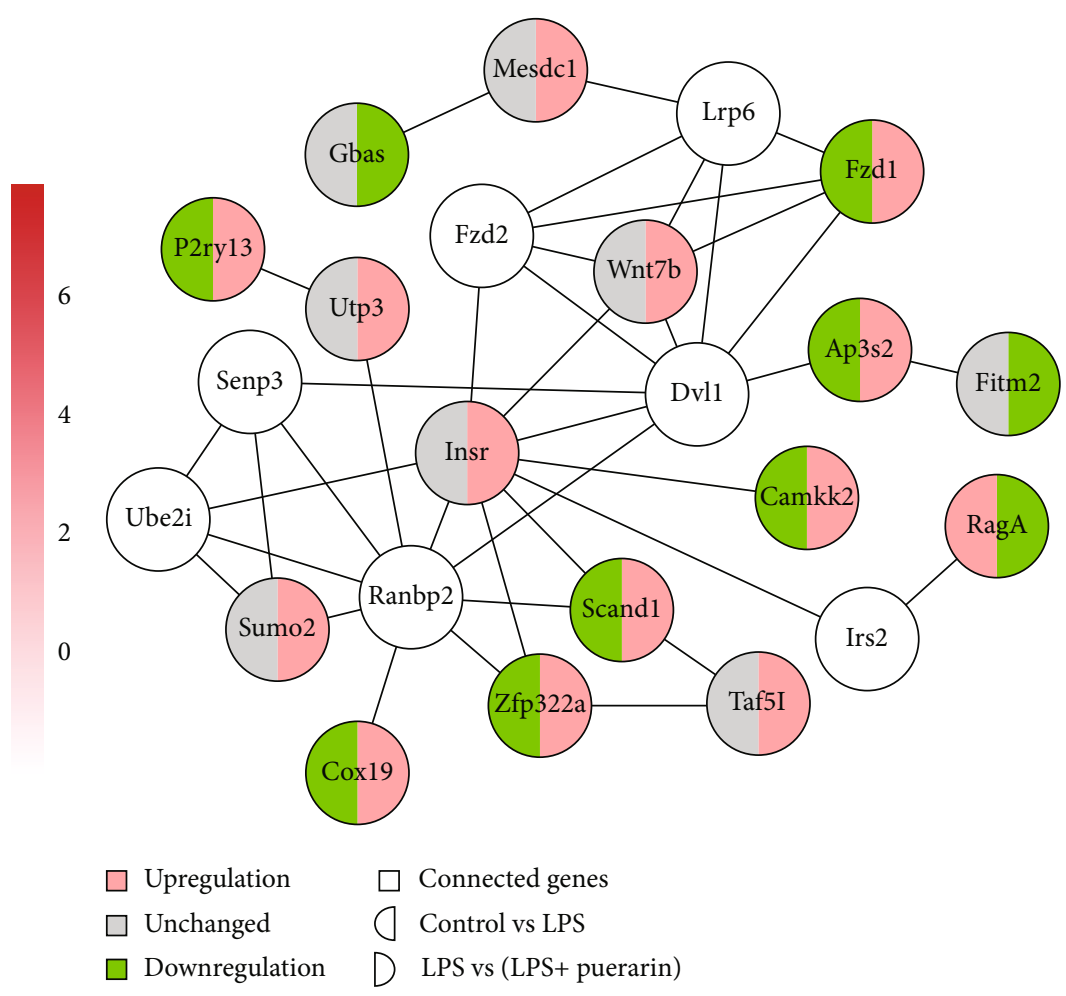

(b)

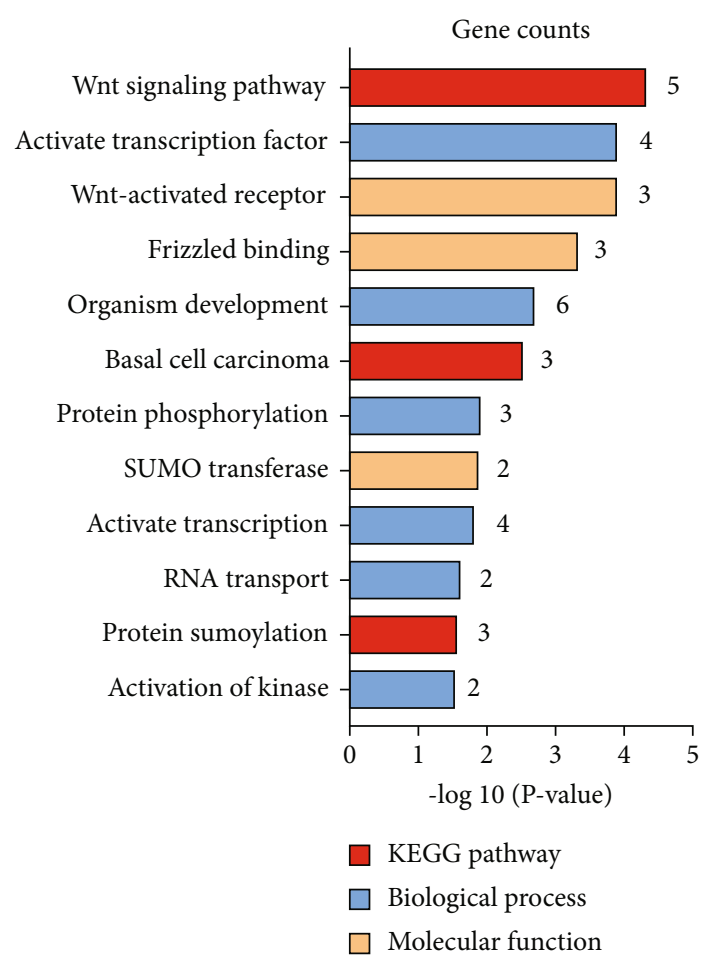

(c)

FIGURE 2: Puerarin significantly affected 16 differentiated genes in LPS-challenged mice. (a) Heatmap of differentially expressed genes. The frontal cortex tissues were collected from 3 groups of mice (i.e., control, LPS, and LPS+puerarin $(60 \mathrm{mg} / \mathrm{kg}), n=3)$ and profiled by nextgeneration RNA sequencing technology. The differentially expressed genes were identified by RNA sequencing and bioinformatics analysis. The heatmap of 16 differentially expressed genes was visualized with $\mathrm{R}$ language. (b) Interaction network of differentially expressed genes. The interaction network of 16 differentially expressed genes and 7 unchanged genes was generated by Cytoscape. The downregulated genes were described as green whereas the upregulated genes were described as red. (c) GO and KEGG pathway analysis of differentially expressed genes. Sixteen differentially expressed genes were analysed for GO and KEGG pathway by DAVID 6.8. 


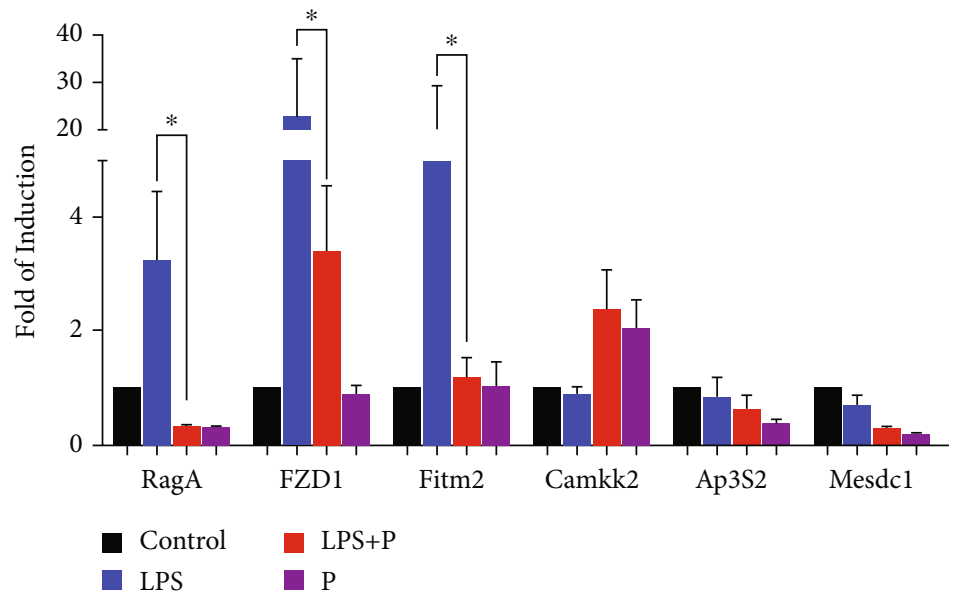

(a)

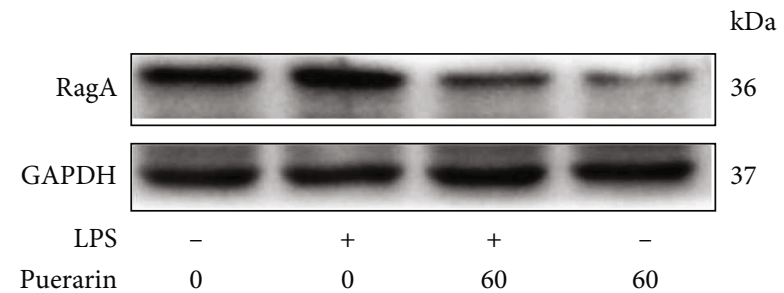

(b)

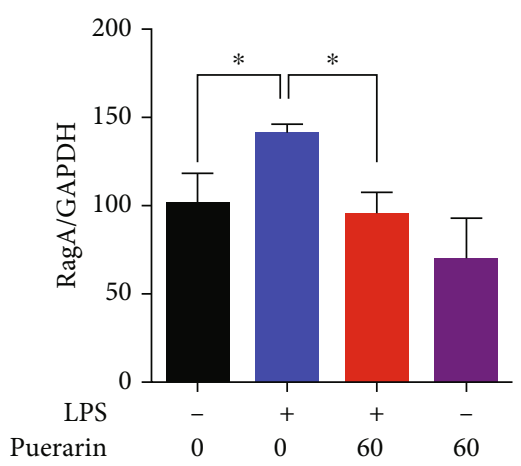

(c)

Figure 3: Continued. 


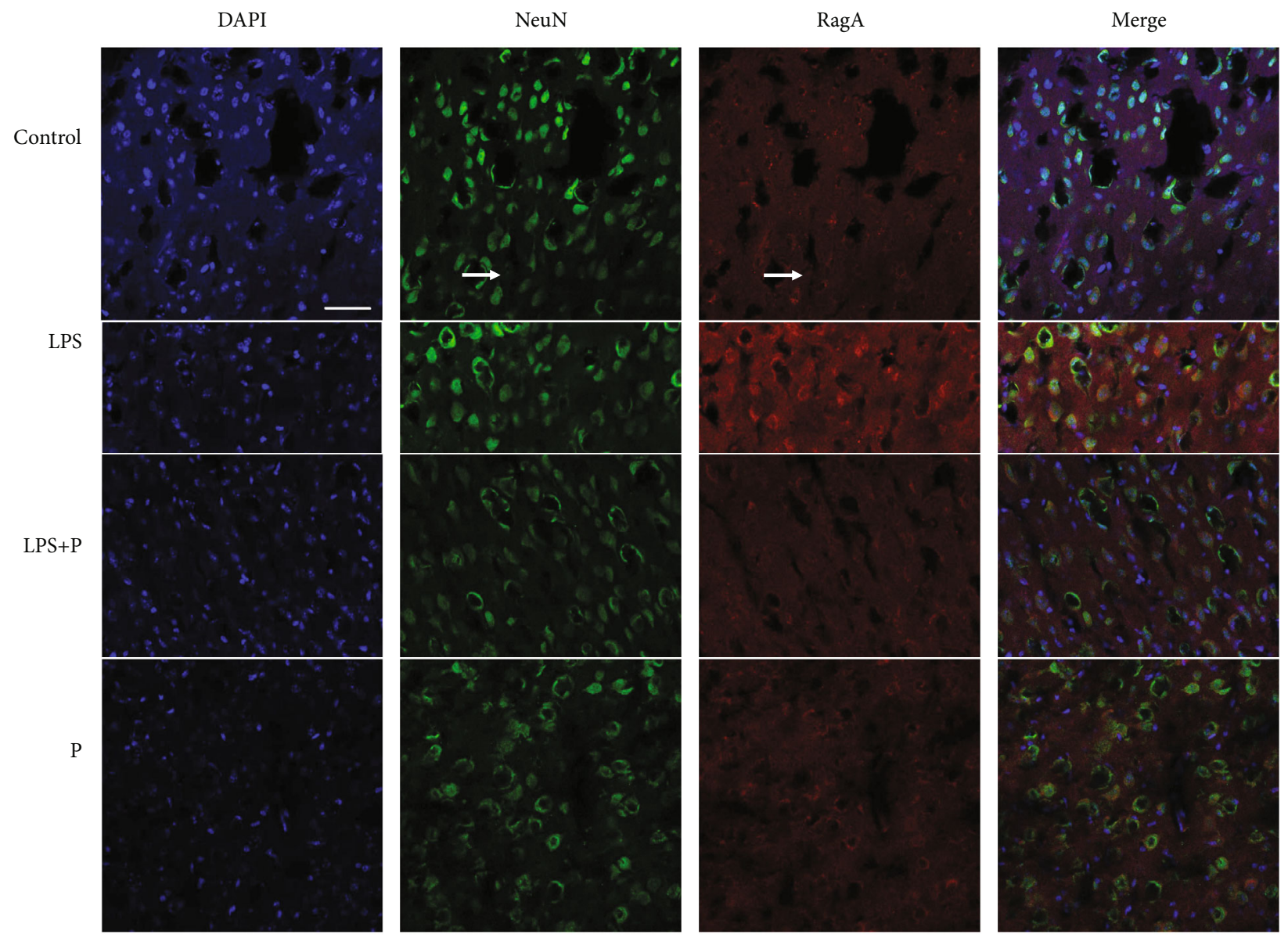

(d)

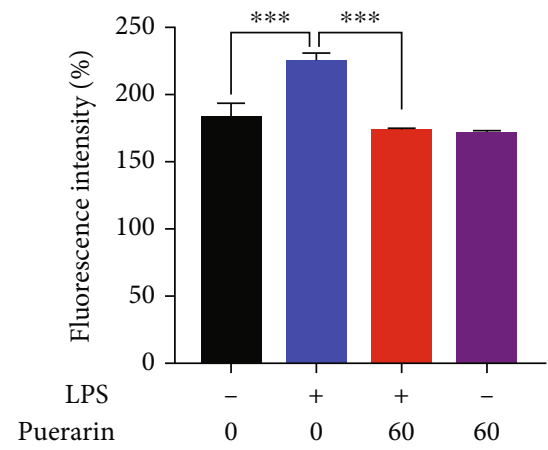

(e)

FIGURE 3: Puerarin downregulated RagA expression in LPS-challenged mice. (a) qRT-PCR quantification of 6 representative differentially expressed genes. The frontal cortex tissues were collected from 4 groups of mice (i.e., control, LPS, LPS + puerarin $(60 \mathrm{mg} / \mathrm{kg})$, and puerarin $(60 \mathrm{mg} / \mathrm{kg}), n=3)$ and analysed by qPCR for selected genes. The data were presented as means \pm SEM $(n=3) .{ }^{*} p<0.05$. (b) Western blot analysis of the protein expression of RagA. The frontal cortex tissues were collected from the experimental mice $(n=3)$ for Western blot analysis of RagA. (c) Quantification of RagA expression. The blots were detected by a densitometric method $(n=3) .{ }^{*} p<$ 0.05. (d) Immunofluorescence detection of RagA expression in the frontal cortex. The frontal cortex tissues were collected from the experimental mice $(n=3)$ for immunohistochemical analysis for RagA expression, whereas DAPI was used to stain nuclear. NeuN was detected as the biomarker for the mature neurons. The images were captured with a Carl Zeiss 700 confocal fluorescence microscope (Jena, Germany). Scale bar, $50 \mu \mathrm{m}$. (e) Quantification of RagA expression. Fluorescence intensity of RagA in (d) was evaluated through the densitometric method $(n=3) .{ }^{* * *} p<0.001$.

and citalopram achieved similar effects on the depressive behaviors and pain in mice whereas ibuprofen appeared to be less effective. In fact, others also evaluated the antidepres- sant effects of puerarin in the other animal models [40]. In this study, we firstly validated that puerarin could attenuate the LPS-induced depressive-like behaviors in mice 


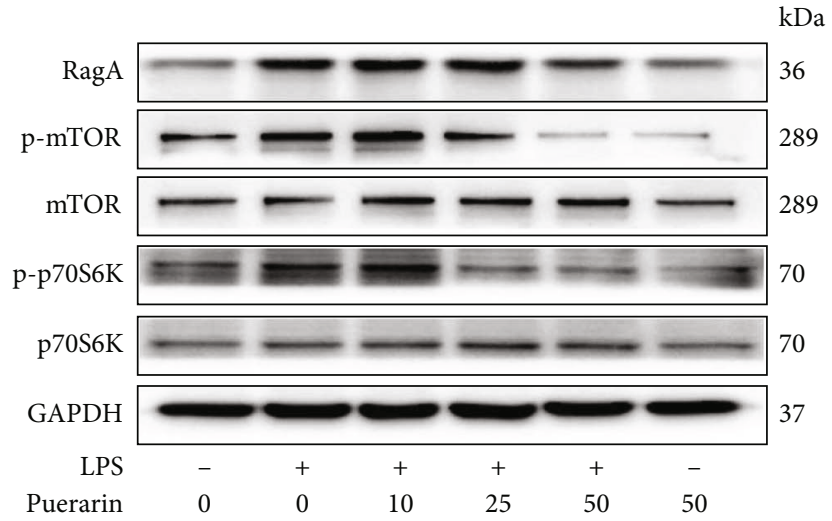

(a)

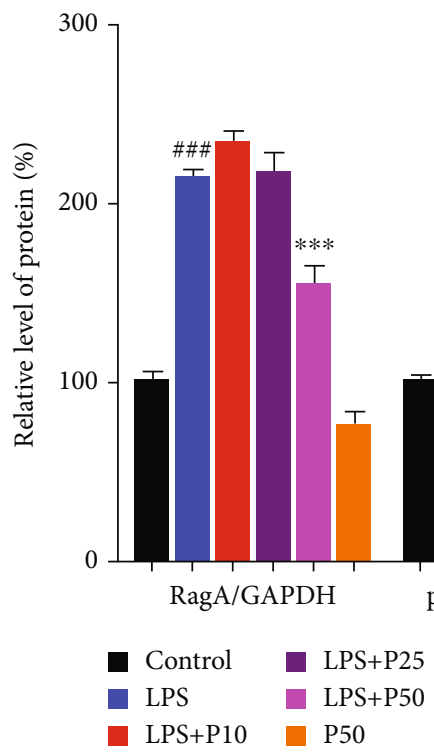

(b)

FIgUre 4: Continued. 

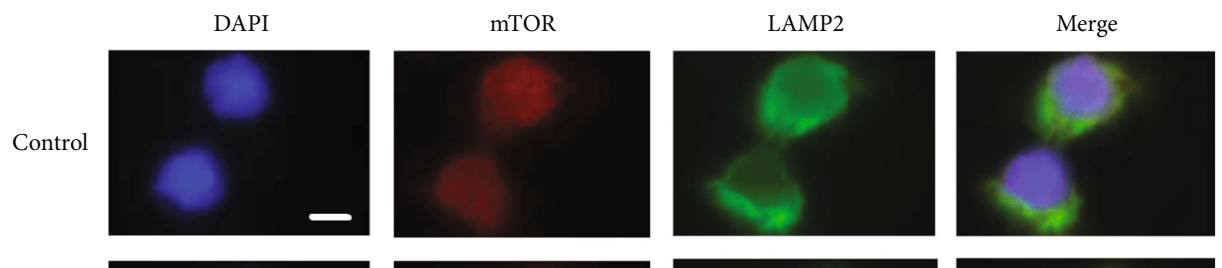

LPS
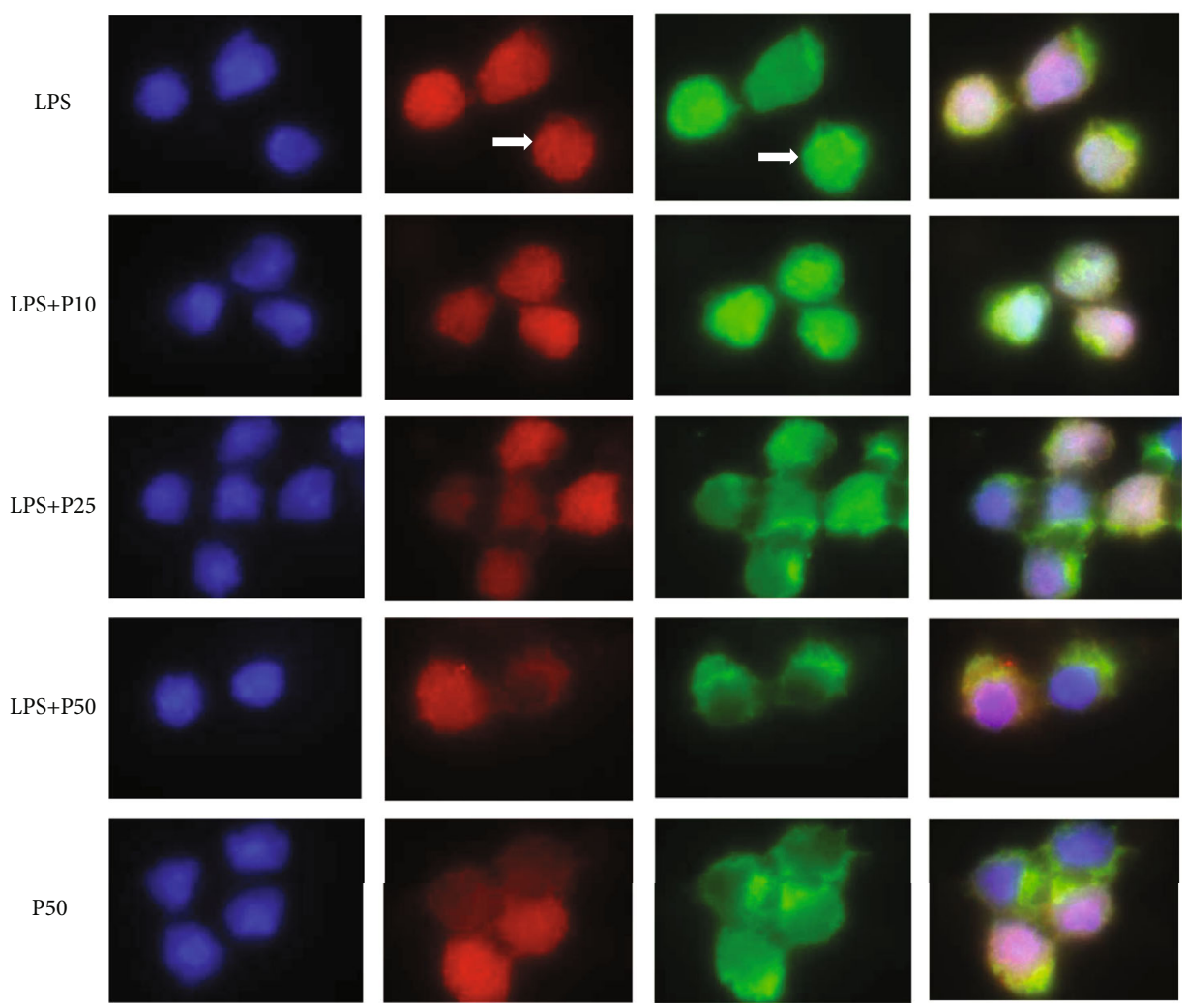

(c)

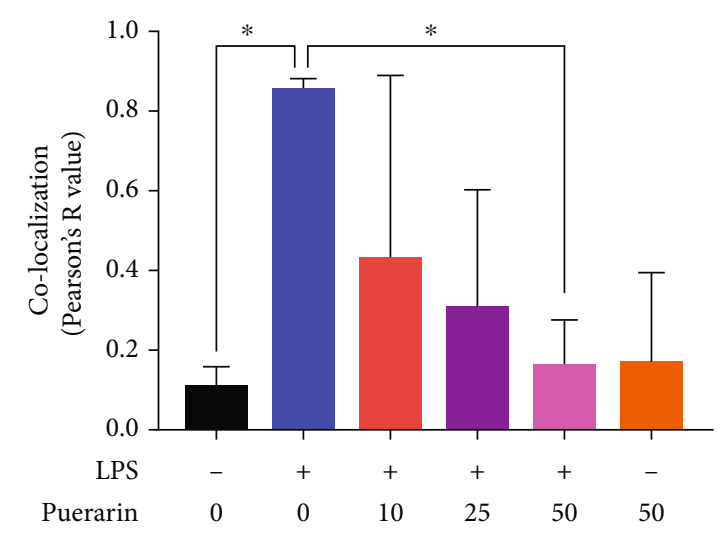

(d)

Figure 4: Puerarin inhibited the RagA/mTOR/p70S6K pathway and the lysosomal localization of mTORC1 in the NSCs. (a) Detection of RagA, mTOR, and p70S6K. The protein expression of RagA, mTOR, and p70S6K were analyzed by Western blot in LPS-challenged neural stem cells. After $24 \mathrm{~h}$ treatment with $200 \mathrm{ng} / \mathrm{ml}$ LPS alone or in combination with puerarin (P) (10, 25, and $50 \mu \mathrm{M})$, NSCs were analyzed by Western blot for the protein expression of RagA, phospho-mTOR (p-mTOR), mTOR, phospho-p70S6K (p-p70S6K), and p70S6K. (b) Quantitation of the blots in (a). The blots in (a) were quantified by the densitometric method. The protein value was shown as means \pm $\mathrm{SD}(n=3) .{ }^{\# \#} p<0.001$ (LPS vs. control); ${ }^{* * *} p<0.001$ (LPS+P vs. LPS). (c) Immunofluorescence staining of mTORC1 in LPSstimulated neural stem cells. After $24 \mathrm{~h}$ treatment with $200 \mathrm{ng} / \mathrm{ml}$ LPS or in combination with P (10, 25, and 50 $\mu$ M), NSCs were incubated with mTOR and lysosomal biomarker LAMP2 antibodies, and DAPI was used to stain nuclear. The images were captured with a Zeiss fluorescence microscope (Jena, Germany). Scale bar, $20 \mu \mathrm{m}$. (d) Quantification of mTOR and LAMP2. Colocalization of mTOR and LAMP2 was quantified by Pearson's $R$ value. The results were shown as means \pm SD $(n=3) .{ }^{*} p<0.05$. 


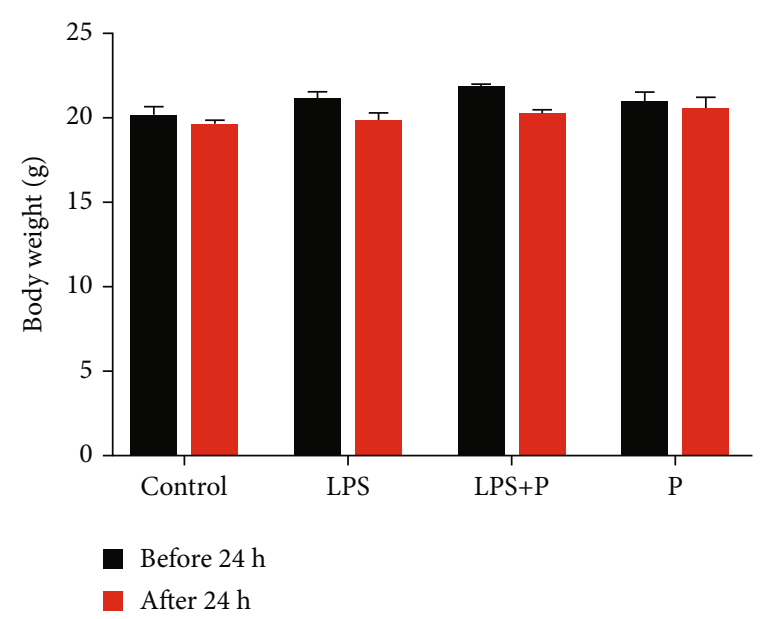

(a)

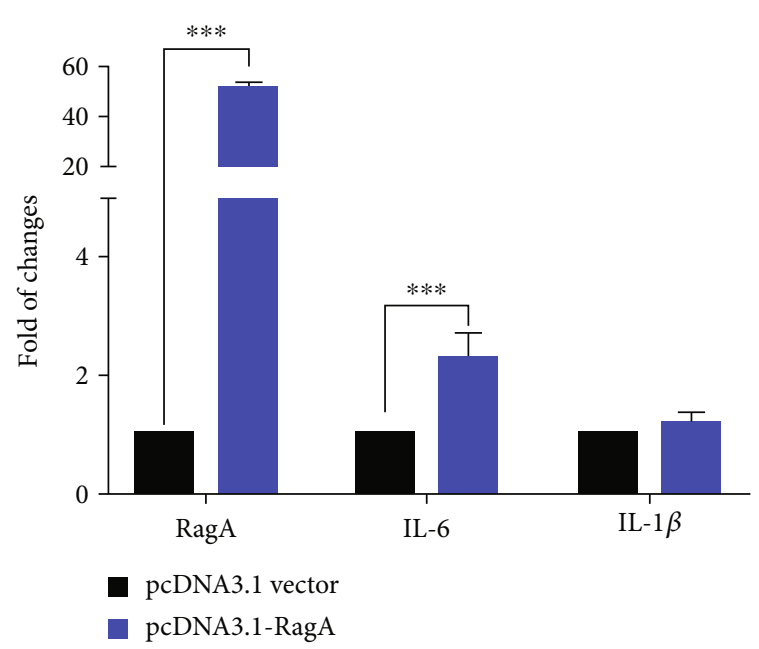

(c)

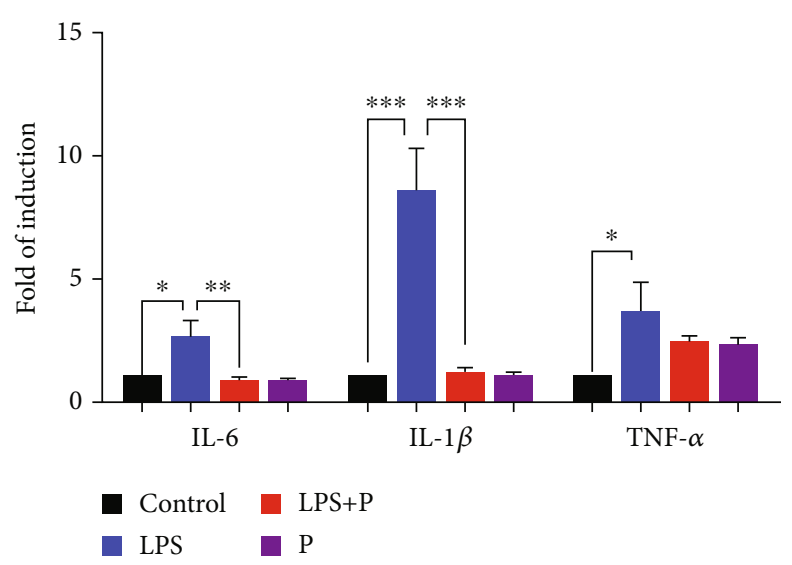

(b)

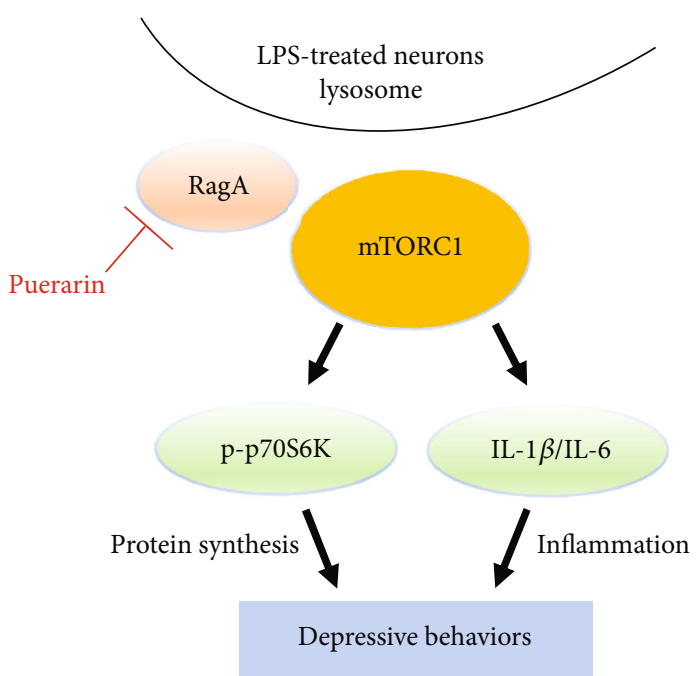

(d)

FIGURE 5: Puerarin attenuated the expression of inflammatory cytokines against LPS stimulation. (a) Body weight of experimental mice. The body weight of the mice in the indicated groups was measured before and after LPS treatment. (b) qRT-PCR quantification of representative inflammatory cytokines. The frontal cortex tissues were collected from 4 groups of mice (i.e., control, LPS, and LPS+puerarin (60 mg/kg), puerarin $(60 \mathrm{mg} / \mathrm{kg}), n=3)$ and analysed by qPCR analysis. The results were shown as means $\pm \operatorname{SEM}(n=3) .{ }^{*} p<0.05 ;{ }^{* *} p<0.01 ;{ }^{* * *} p$ $<0.001$. (c) qRT-PCR quantification of RagA, IL-6, and IL-1 $\beta$. Highly differentiated PC12 cells were transfected with pcDNA3.1 vector or pcDNA3.1-RagA plasmid for $24 \mathrm{~h}$. The cells were collected for qPCR analysis. The data were shown as means \pm SD $(n=3)$. ${ }^{* * *} p<$ 0.001. (d) The diagram illustrating the potential mechanism. Puerarin targeted RagA and thereby inhibited the mTOR/p70S6K pathway and suppressed the mRNA levels of proinflammatory cytokines (e.g., IL-6 and IL-1 $\beta$ ) towards the amelioration of depressive behaviors.

(Figures 1(c) and 1(d)). Subsequently, we focused on the molecular mechanisms underlying the antidepressant activity of puerarin. Our strategy was to identify differentially expressed genes in mice after treatment with LPS and puerarin, alone or in combination, by next-generation RNA sequencing technology. Indeed, we found that puerarin downregulated the expression of RagA mRNA by 60 folds compared with LPS-stimulated group. We further investigated the effects of puerarin on RagA/mTOR/p70S6K pathway, the accumulation of mTORC1 at the lysosomal membrane, and the production of proinflammatory cytokines.

Next-generation RNA sequencing technology is widely used to profile the transcriptomes of cultured cells and ani- mal models for differentially expressed genes [41]. In our study, adult male mice were randomly divided into four groups: control, LPS, LPS+Puearin, and puearin. RNA sequencing profiles revealed that puerarin affected RagA and other 15 genes against LPS stimulation (Figures 2(a) and 2(b)). The functional annotation classified these genes into multicellular organism development, small ubiquitinlike modifier (SUMO) transferase activity, activation of protein kinase activity, DNA-binding transcription factor activity, protein heterodimerization activity, and other activities (Figure 2(c)). As for multicellular organism development, puerarin upregulated small subunit processome component (Utp3) and downregulated Wnt family member 7B (Wnt7b) and frizzled class receptor 1 (Fzd1). Utp3 is involved in the 
development of the osteosarcoma through regulating the ribosome biogenesis [42]. Wnt7b is a key ligand for the Wnt signaling pathway and mainly regulates the angiogenesis in the brain and spinal cord [43]. Fzd1 is a receptor for the Wnt signaling and regulates the adult hippocampal neurogenesis [44]. For SUMO transferase activity, puerarin upregulated the isoform Sumo2. Sumo2 is directly involved in the protein sumoylation for controlling the cognitive process in mice [45]. For the activation of protein kinase activity, puerarin upregulated insulin receptor (Insr) and calcium/calmodulin-dependent protein kinase kinase 2 (Camkk2). Insr refers to a tyrosine kinase receptor and transmitted the ligands of insulin to bind to the intracellular signaling [46]. Insr manifested as isoform A and isoform B. Insr-A was mainly expressed in the brain and uniquely expressed in the neuron. Insr-B was mainly expressed in the peripheral tissue [47]. Insr is upregulated in the entorhinal cortex and hippocampus in patients with Alzheimer's disease compared with elderly control group [48]. Camkk2 is characterized as a protein kinase for regulating cell survival and proliferation [49]. Camkk2 could decrease the expression of proinflammatory cytokines and promote LPS-treated primary microglial cells to undergo M2 polarization [50]. For protein transporter activity, puerarin upregulated adaptor-related protein complex 3 subunit sigma 2 (AP3S2). Previous study identified that some AP3S2 genetic variants are related to the onset of type-2 diabetes [51]. For DNA-binding transcription factor activity, puerarin upregulated SCAN domain containing 1 (Scand1), TATA-boxbinding protein-associated factor 5 like (Taf5l), and zinc finger protein 322 (Zfp322a). Scand1 is suggested to be a potential druggable target in the treatment of anxiety disorder [52]. Scand1 is upregulated by carbon monoxide in the cortical astrocytes [53]. Taf5l is a component of the P300/CBPassociated factor (PCAF) complex with the activity to acetylate the histones [54]. Another study showed that Taf5l is an epigenetic regulator for mediating the mouse embryonic stem cell state [55]. Zfp322a regulates the pluripotency of the mouse embryonic stem cells and increases the reprogramming efficiency [56]. For protein heterodimerization activity, puerarin downregulated small GTPase RagA. RagA senses amino acids for the mTORC1 signaling pathway involving in cell growth and metabolism [14]. For other activities, puerarin downregulated cytochrome c oxidase 19 (Cox19), fat storage-inducing transmembrane protein 2 (Fitm2), and glioblastoma amplified sequence (Gbas). Cox19 is involved in the copper efflux to the mitochondria [57]. Fitm 2 regulates in the normal fat storage in adipose tissues in mice and plays critical role in the cell homeostasis and the locomotor functions [58]. Gbas was expressed in the brain and heart to regulate the CREB signaling pathway, $\mathrm{Ca}^{2+}$ influx, and vesicular transport [59]. On the other hand, puerarin upregulated mesoderm development candidate 1 (Mesdc1) and purinergic receptor P2Y13 (P2ry13). Mesdc1 is an actin-binding protein and has an oncogenicity in the bladder cancer and hepatocellular carcinoma [60]. P2ry13 is mainly expressed in the microglia, not neuron or astrocyte. Interestingly, P2ry13 disruption enhances the proliferation of progenitor cells and the formation of new neurons
[61]. Based on the gene abundance, fold changes and biological relevance, RagA was selected for further study in current study.

Our group recently revealed that LPS markedly upregulated RagA expression and activated mTORC1 pathway [8]. RagA forms heterodimers with other isoforms for different functions, for example, RagA/RagB heterodimer for binding GTP and RagC/RagD heterodimer for binding GDP [62]. Importantly, RagA senses the cellular sufficiency of amino acids for the mTOR signaling pathway [63]. When amino acids are deficient, the inactive heterodimer (RagA/B) recruits the trimeric tuberous sclerosis complex (TSC) to the lysosome, thereby preventing the association with mTORC1 activator Ras-homolog accumulated in brain (Rheb) [64]. When the amino acids are sufficient, however, heterodimer RagA/B is loaded with GTP to bind the regulatoryassociated protein of mammalian target of rapamycin (Raptor), recruit mTORC1 to the lysosome surface to form complex with Rheb and activate mTORC1 [65]. The effect of mTORC1 signaling pathway is the regulation of cell growth and metabolism while dysregulation of mTORC1 is implicated in the neurodegenerative diseases, cancer, and diabetes [66]. The phosphorylation of the downstream targets including p70S6K and 4EBP1 exhibit the activity of mTORC1 [67]. Phosphorylated p70S6K promotes the protein synthesis. Phosphorylated 4EBP1 does not bind to the eukaryotic translation initiation factor $4 \mathrm{E}$ (EIF4E), which promotes the cap-dependent mRNA translation [68]. The p70S6K signaling pathway is a potential mechanism mediating the major depressive disorder [69]. High-fat diet caused the anxiety and anhedonia via reducing the expression of phosphoP70S6K in the frontal cortex of rats [70]. In the present study, RagA, FZD1, Fitm2, Camkk2, Ap3S2, and Mesdc1 were selected for qPCR validation. LPS increased the mRNA levels of RagA, FZD1, and Fitm2 compared with the control group. Puerarin at the dosage of $60 \mathrm{mg} / \mathrm{kg}$ significantly reduced the expression of mRNA levels of RagA, FZD1, and Fitm2 against LPS stimulation although the mRNA levels of Camkk2, Ap3S2, and Mesdc1 remained unchanged (Figure 3(a)). Puerarin effectively reduced RagA in LPSchallenged mice (Figures $3(\mathrm{~b})-3(\mathrm{e})$ ). Such results appeared to be consistent with the results of RNA-seq profiling. Neurogenesis is implicated in the depression [71]. Our previous study demonstrated that LPS activated the RagA/mTOR/p70S6K pathway and caused the lysosomal accumulation of mTORC1 in the NSCs [8]. In our study, we validated that puerarin inhibited the $\mathrm{RagA} / \mathrm{mTOR} / \mathrm{p} 70 \mathrm{~S} 6 \mathrm{~K}$ pathway and reduced the accumulation of mTORC1 lysosomal in neural stem cells (Figures 4(a)-4(d)). Moreover, LPS is well known to induce inflammation in mice [37]. In this study, we firstly confirmed that LPS and puerarin could not cause significant sickness in mice (Figure 5(a)). Such results clarified that depressive behaviors were not resulted from LPSinduced sickness. Secondly, we examined the effects of puerarin on proinflammatory cytokines. Indeed, puerarin effectively reduced corresponding mRNA level of IL- 6 and IL$1 \beta$ and affected TNF- $\alpha$ to a lesser extent in LPS-challenged mice (Figure 5(b)). As for the potential role of RagA in LPS-induced inflammatory response, we examined whether 
ectopic expression of RagA could influence the expression of proinflammatory cytokines (e.g., IL-6 and IL-1 $\beta$ ). As result, the overexpression of RagA led to the significant increase in IL-6 mRNA levels, but not IL- $1 \beta$ mRNA in highly differentiated PC12 cells (Figure 5(c)). Interestingly, mTORC1 often cross-talks with $\mathrm{mTORC} 2$ to regulate the production of different cytokines [72]. Combining mTORC1/mTORC2 with pp242 or high-dose rapamycin upregulated IL-1 $\beta$ and TNF- $\alpha$ but downregulated IL-6 [73]. In addition, the mTORC1 pathway regulated IL- $\beta$ by dendritic cells [74]. Presumably, RagA links the mTORC1 signaling pathway to the expression of different proinflammatory cytokines in a cell context specific manner. Taken together, puerarin inhibited the RagA/mTOR/p70S6K pathway and reduced the production of proinflammatory cytokines (e.g., IL-6, IL-1 $\beta$ ) against LPS stimulation. Ultimately, puerarin could effectively ameliorate LPS-induced depressive behaviors (Figure 5(d)).

\section{Conclusions}

The results from the present study not only validated that botanical drug puerarin could attenuate LPS-stimulated depressive-like behaviors in mice but also revealed that the inhibition of $\mathrm{RagA} / \mathrm{mTOR} / \mathrm{p} 70 \mathrm{~S} 6 \mathrm{~K}$ pathways could be a potential antidepressant mechanism. At the molecular level, puerarin downregulated RagA expression, reduced the lysosomal translocation of mTORC1, and inhibited the activation of mTOR/p70S6K pathway. Consequently, puerarin markedly reduced the expression of proinflammatory cytokines, especially, IL-6, in LPS-challenged mice. Thus, puerarin may be a promising antidepressant and antiinflammatory candidate drug for the alleviation of depression in $\mathrm{AD}$ and other diseases.

\section{Abbreviations}

$\begin{array}{ll}\text { LPS: } & \text { Liposaccharide } \\ \text { RagA: } & \text { Ras-related GTP binding protein A } \\ \text { qRT-PCR: } & \text { Quantitative real-time PCR } \\ \text { AD: } & \text { Alzheimer's disease } \\ \text { IL: } & \text { Interleukin } \\ \text { GTPases: } & \text { Guanosine triphosphatases } \\ \text { mTOR: } & \text { Mammalian target of rapamycin } \\ \text { GTP: } & \text { Guanosine triphosphate } \\ \text { GDP: } & \text { Guanosine diphosphate } \\ \text { LAMP2: } & \text { Lysosome-associated membrane protein } 2 \\ \text { p70S6K: } & \text { p70 S6 kinase } \\ \text { 4E-BP1: } & \text { 4E-binding protein 1 } \\ \text { CUMS: } & \text { Chronic unpredictable mild stress } \\ \text { DMEM: } & \text { Dulbecco's modified Eagle's medium } \\ \text { FBS: } & \text { Fetal bovine serum } \\ \text { HS: } & \text { Horse serum } \\ \text { bFGF: } & \text { Basic fibroblast growth factor } \\ \text { EGF: } & \text { Epidermal growth factor } \\ \text { TST: } & \text { Tail suspension test } \\ \text { FST: } & \text { Forced swim test } \\ \text { FC: } & \text { Fold change } \\ \text { KEGG: } & \text { Kyoto Encyclopedia of Genes and Genomes }\end{array}$

$\begin{array}{ll}\text { GO: } & \text { Gene Ontology } \\ \text { PCR: } & \text { Polymerase chain reaction } \\ \text { NSC: } & \text { Neural stem cell } \\ \text { PFA: } & \text { Paraformaldehyde } \\ \text { PBS: } & \text { Phosphate-buffered saline } \\ \text { RT: } & \text { Room temperature } \\ \text { DAPI: } & 4^{\prime} \text {-6-diamidino-2-phenylindole } \\ \text { ANOVA: } & \text { One-way analysis of variance } \\ \text { Utp3: } & \text { Small subunit processome component } \\ \text { Wnt7b: } & \text { Wnt family member 7B } \\ \text { Fzd1: } & \text { Frizzled class receptor 1 } \\ \text { Sumo2: } & \text { Small ubiquitin-like modifier } 2 \\ \text { Insr: } & \text { Insulin receptor } \\ \text { Camkk2: } & \text { Calcium/calmodulin-dependent protein kinase } \\ & \text { kinase } 2 \\ \text { AP3S2: } & \text { Adaptor-related protein complex } 3 \text { subunit } \\ & \text { sigma 2 } \\ \text { Scand1: } & \text { SCAN domain containing 1 } \\ \text { Taf5l: } & \text { TATA-box-binding protein-associated factor } 5 \\ & \text { like } \\ \text { Zfp322a: } & \text { Zinc finger protein 322 } \\ \text { PCAF: } & \text { P300/CBP-associated factor } \\ \text { Cox19: } & \text { Cytochrome c oxidase 19 } \\ \text { Fitm2: } & \text { Fat storage-inducing transmembrane protein } 2 \\ \text { Gbas: } & \text { Glioblastoma amplified sequence } \\ \text { Mesdc1: } & \text { Mesoderm development candidate 1 } \\ \text { P2ry13: } & \text { Purinergic receptor P2Y13 } \\ \text { TSC: } & \text { Tuberous sclerosis complex } \\ \text { Rheb: } & \text { Ras-homolog enriched in brain } \\ \text { Raptor: } & \text { Regulatory-associated protein of mammalian } \\ & \text { target of rapamycin } \\ \text { EIF4E: } & \text { Eukaryotic translation initiation factor } 4 \text { E. } \\ & \end{array}$

\section{Data Availability}

The data that support the findings of this study were available from the corresponding author upon reasonable request.

\section{Ethical Approval}

Animal experiments were approved by the Committee on the Use of Live Animals in Teaching and Research of the University of Hong Kong (CULATR 4284-17).

\section{Conflicts of Interest}

The authors demonstrate that there is no conflict of interest.

\section{Authors' Contributions}

Jia Zhao, Wei Zhao, Huixin Chen, Xiuying Zhang, and Dan Luo performed the experiments; Yizhen Jia, Fung Yin Ngo, and Youqiang Song analyzed the data of RNA sequencing. Lixing Lao supervised the data analysis; Jianhui Rong designed the research and revised the article. 


\section{Acknowledgments}

Sincere thanks to Mr. Wong Hei Kiu, Ms. Lee Wai Sin, and Mr. Shek Chun Shum for their professional technical assistance. The computing operation were carried out using research computing facilities offered by Information Technology Services, the University of Hong Kong. This work was supported by National Natural Science Foundation of China (Nos. 21778046 and 81703726), the Health and Medical Research Fund (HMRF 15161731, 16171751, and 17181231), General Research Fund (GRF) grants (17120915, 17146216, 17100317, and 17119619), Research and Cultivation Plan of High-Level Hospital Construction (HKUSZH201902040), Science, Technology and Innovation Commission of Shenzhen Municipality (JCYJ20180306173835901), and Midstream Research Programme for Universities (MRP) 053/18X.

\section{References}

[1] V. Orgeta, N. Tabet, R. Nilforooshan, and R. Howard, "Efficacy of antidepressants for depression in Alzheimer's disease: systematic review and meta-analysis," Journal of Alzheimer's Disease, vol. 58, no. 3, pp. 725-733, 2017.

[2] M. E. Fox and M. K. Lobo, "The molecular and cellular mechanisms of depression: a focus on reward circuitry," Molecular Psychiatry, vol. 24, no. 12, pp. 1798-1815, 2019.

[3] L. Leng, K. Zhuang, Z. Liu et al., "Menin deficiency leads to depressive-like behaviors in mice by modulating astrocytemediated neuroinflammation," Neuron, vol. 100, no. 3, pp. 551-563.e7, 2018, e7.

[4] M. L. Wong, A. Inserra, M. D. Lewis et al., "Inflammasome signaling affects anxiety- and depressive-like behavior and gut microbiome composition," Molecular Psychiatry, vol. 21, no. 6, pp. 797-805, 2016.

[5] E. G. Vichaya, G. Laumet, D. L. Christian et al., "Motivational changes that develop in a mouse model of inflammationinduced depression are independent of indoleamine 2,3 dioxygenase," Neuropsychopharmacology, vol. 44, no. 2, pp. 364$371,2019$.

[6] C. Barua, B. Saikia, X. Ren et al., "Zanthoxylum alatum attenuates lipopolysaccharide-induced depressive-like behavior in mice hippocampus," Pharmacognosy Magazine, vol. 14, no. 59, p. 673, 2018.

[7] C. C. S. K. Barua, P. Haloi, L. Buragohain et al., "Erigeron linifolius attenuates lipopolysachharide-induced depressive-like behavior in mice by impeding neuroinflammation, oxidonitrosative stress, and upregulation of tropomyosin receptor kinase B-derived neurotrophic factor and monoaminergic pathway in the hippocampus," Pharmacognosy Magazine, vol. 15, no. 62, p. 12, 2019.

[8] J. Zhao, L. X. Lao, W. Cui, and J. H. Rong, "Potential link between the RagA-mTOR-p70S6K axis and depressivebehaviors during bacterial liposaccharide challenge," Journal of Neuroinflammation, vol. 16, no. 1, 2019.

[9] J. O. Lipton and M. Sahin, “The neurology of mTOR," Neuron, vol. 84, no. 2, pp. 275-291, 2014.

[10] D. W. Lamming, L. Ye, P. Katajisto et al., "Rapamycin-induced insulin resistance is mediated by mTORC2 loss and uncoupled from longevity," Science, vol. 335, no. 6076, pp. 1638-1643, 2012.
[11] J. Kim and K. L. Guan, "mTOR as a central hub of nutrient signalling and cell growth," Nature Cell Biology, vol. 21, no. 1, pp. 63-71, 2019.

[12] A. Ardestani, B. Lupse, Y. Kido, G. Leibowitz, and K. Maedler, "mTORC1 Signaling: A Double-Edged Sword in Diabetic $\beta$ Cells," Cell Metabolism, vol. 27, no. 2, pp. 314-331, 2018.

[13] X. Gu, J. M. Orozco, R. A. Saxton et al., "SAMTOR is anSadenosylmethionine sensor for the mTORC1 pathway," Science, vol. 358, no. 6364, pp. 813-818, 2017.

[14] R. L. Wolfson and D. M. Sabatini, "The dawn of the age of amino acid sensors for the mTORC1 pathway," Cell Metabolism, vol. 26, no. 2, pp. 301-309, 2017.

[15] R. L. Wolfson, L. Chantranupong, G. A. Wyant et al., "KICSTOR recruits GATOR1 to the lysosome and is necessary for nutrients to regulate mTORC1," Nature, vol. 543, no. 7645, pp. 438-442, 2017.

[16] C. M. Hale, Q. Cheng, D. Ortuno et al., "Identification of modulators of autophagic flux in an image-based high content siRNA screen," Autophagy, vol. 12, no. 4, pp. 713-726, 2016.

[17] S. Notomi, K. Ishihara, N. E. Efstathiou et al., "Genetic LAMP2 deficiency accelerates the age-associated formation of basal laminar deposits in the retina," Proceedings of the National Academy of Sciences of the United States of America, vol. 116, no. 47, pp. 23724-23734, 2019.

[18] K. Fukumoto, M. V. Fogaça, R. J. Liu et al., "Activity-dependent brain-derived neurotrophic factor signaling is required for the antidepressant actions of $(2 \mathrm{R}, 6 \mathrm{R})$-hydroxynorketamine," Proceedings of the National Academy of Sciences of the United States of America, vol. 116, no. 1, pp. 297-302, 2019.

[19] A. Aguilar-Valles, D. de Gregorio, E. Matta-Camacho et al., "Antidepressant actions of ketamine engage cell-specific translation via eIF4E," Nature, vol. 590, no. 7845, pp. 315-319, 2021.

[20] L. M. Monteggia, R. C. Malenka, and K. Deisseroth, “The best way forward," Nature, vol. 515, no. 7526, pp. 200-201, 2014.

[21] Y. Liu, D. Lin, B. Wu, and W. Zhou, "Ketamine abuse potential and use disorder," Brain Research Bulletin, vol. 126, Part 1, pp. 68-73, 2016.

[22] X. Sun, T. Zhang, Y. Zhao, E. Cai, H. Zhu, and S. Liu, "The protective effect of 5-O-methylvisammioside on LPS-induced depression in mice by inhibiting the over activation of BV-2 microglia through Nf- $\kappa \mathrm{B} / \mathrm{I} \kappa \mathrm{B}-\alpha$ pathway," Phytomedicine, vol. 79, article 153348, 2020.

[23] J. Zhao, D. Luo, Z. Liang, L. Lao, and J. Rong, "Plant natural product puerarin ameliorates depressive behaviors and chronic pain in mice with spared nerve injury (SNI)," Molecular Neurobiology, vol. 54, no. 4, pp. 2801-2812, 2017.

[24] X. Song, W. Wang, S. Ding, X. Liu, Y. Wang, and H. Ma, "Puerarin ameliorates depression-like behaviors of with chronic unpredictable mild stress mice by remodeling their gut microbiota," Journal of Affective Disorders, vol. 290, pp. 353-363, 2021.

[25] A. Tantipongpiradet, O. Monthakantirat, O. Vipatpakpaiboon et al., "Effects of puerarin on the ovariectomy-induced depressive-like behavior in ICR mice and its possible mechanism of action," Molecules, vol. 24, no. 24, p. 4569, 2019.

[26] J. L. Warner-Schmidt, K. E. Vanover, E. Y. Chen, J. J. Marshall, and P. Greengard, "Antidepressant effects of selective serotonin reuptake inhibitors (SSRIs) are attenuated by antiinflammatory drugs in mice and humans," Proceedings of the 
National Academy of Sciences of the United States of America, vol. 108, no. 22, pp. 9262-9267, 2011.

[27] K. F. Hansen, K. Sakamoto, C. Pelz, S. Impey, and K. Obrietan, "Profiling status epilepticus-induced changes in hippocampal RNA expression using high-throughput RNA sequencing," Scientific Reports, vol. 4, no. 1, 2015.

[28] R. C. Bagot, H. M. Cates, I. Purushothaman et al., "Circuitwide transcriptional profiling reveals brain region-specific gene networks regulating depression susceptibility," Neuron, vol. 90, no. 5, pp. 969-983, 2016.

[29] M. Pertea, D. Kim, G. M. Pertea, J. T. Leek, and S. L. Salzberg, "Transcript-level expression analysis of RNA-seq experiments with HISAT, StringTie and Ballgown," Nature Protocols, vol. 11, no. 9, pp. 1650-1667, 2016.

[30] A. C. Frazee, G. Pertea, A. E. Jaffe, B. Langmead, S. L. Salzberg, and J. T. Leek, "Ballgown bridges the gap between transcriptome assembly and expression analysis," Nature Biotechnology, vol. 33, no. 3, pp. 243-246, 2015.

[31] F. Y. Ngo, W. Wang, Q. Chen et al., "Network Pharmacology Analysis and Molecular Characterization of the Herbal Medicine Formulation Qi-Fu-Yin for the Inhibition of the Neuroinflammatory Biomarker iNOS in Microglial BV-2 Cells: Implication for the Treatment of Alzheimer's Disease," Oxidative Medicine and Cellular Longevity, vol. 2020, Article ID 5780703, 15 pages, 2020.

[32] W. Zhu, J. Li, Y. Liu, K. Xie, L. Wang, and J. Fang, "Mesencephalic astrocyte-derived neurotrophic factor attenuates inflammatory responses in lipopolysaccharide-induced neural stem cells by regulating NF- $\kappa \mathrm{B}$ and phosphorylation of $\mathrm{p} 38$ MAPKs pathways," Immunopharmacology and Immunotoxicology, vol. 38, no. 3, pp. 205-213, 2016.

[33] H. Azari, S. Sharififar, M. Rahman, S. Ansari, and B. A. Reynolds, "Establishing embryonic mouse neural stem cell culture using the neurosphere assay," Journal of Visualized Experiments, vol. 47, no. 47, 2011.

[34] J. Zhao, Y. Cheng, C. Yang et al., "Botanical drug puerarin attenuates 6-hydroxydopamine (6-OHDA)-induced neurotoxicity via upregulating mitochondrial enzyme arginase-2," Molecular Neurobiology, vol. 53, no. 4, pp. 2200-2211, 2016.

[35] D. Luo, Y. Guo, Y. Cheng, J. Zhao, Y. Wang, and J. Rong, "Natural product celastrol suppressed macrophage M1 polarization against inflammation in diet-induced obese mice via regulating Nrf2/HO-1, MAP kinase and NF- $\kappa \mathrm{B}$ pathways," Aging (Albany NY), vol. 9, no. 10, pp. 2069-2082, 2017.

[36] R. J. Elsworthy and S. Aldred, "Depression in Alzheimer's disease: an alternative role for selective serotonin reuptake inhibitors?," Journal of Alzheimer's Disease, vol. 69, no. 3, pp. 651661, 2019.

[37] A. K. Walker, E. E. Wing, W. A. Banks, and R. Dantzer, "Leucine competes with kynurenine for blood-to-brain transport and prevents lipopolysaccharide-induced depression-like behavior in mice," Molecular Psychiatry, vol. 24, no. 10, pp. 1523-1532, 2019.

[38] J. Rothmore, "Antidepressant-induced sexual dysfunction," The Medical Journal of Australia, vol. 212, no. 7, pp. 329334, 2020.

[39] L. T. Park and C. A. Zarate Jr., "Depression in the primary care setting," The New England Journal of Medicine, vol. 380, no. 6, pp. 559-568, 2019.

[40] J. Cheng, M. Chen, J. X. Zhu et al., "FGF-2 signaling activation in the hippocampus contributes to the behavioral and cellular responses to puerarin," Biochemical Pharmacology, vol. 168, pp. 91-99, 2019.

[41] M. J. Girgenti and R. S. Duman, "Transcriptome alterations in posttraumatic stress disorder," Biological Psychiatry, vol. 83, no. 10, pp. 840-848, 2018.

[42] B. Liu, Z. Zhang, E. N. Dai, J. X. Tian, J. Z. Xin, and L. Xu, "Modeling osteosarcoma progression by measuring the connectivity dynamics using an inference of multiple differential modules algorithm," Molecular Medicine Reports, vol. 16, no. 2, pp. 1047-1054, 2017.

[43] C. Cho, P. M. Smallwood, and J. Nathans, "Reck and Gpr124 are essential receptor cofactors for Wnt7a/Wnt7b-specific signaling in mammalian CNS angiogenesis and blood-brain barrier regulation," Neuron, vol. 95, no. 5, pp. 1056-1073.e5, 2017.

[44] M. D. Mardones, G. A. Andaur, M. Varas-Godoy et al., "Frizzled-1 receptor regulates adult hippocampal neurogenesis," Molecular Brain, vol. 9, no. 1, p. 29, 2016.

[45] S. Yu, F. Galeffi, R. M. Rodriguiz et al., "Small ubiquitin-like modifier 2 (SUMO2) is critical for memory processes in mice," The FASEB Journal, vol. 34, no. 11, pp. 14750-14767, 2020.

[46] M. Gralle, "The neuronal insulin receptor in its environment," Journal of Neurochemistry, vol. 140, no. 3, pp. 359-367, 2017.

[47] I. Pomytkin, J. P. Costa-Nunes, V. Kasatkin et al., "Insulin receptor in the brain: mechanisms of activation and the role in the CNS pathology and treatment," CNS Neuroscience \& Therapeutics, vol. 24, no. 9, pp. 763-774, 2018.

[48] I. B. da Costa, R. W. de Labio, L. T. Rasmussen et al., "Change in INSR, APBA2 and IDE gene expressions in brains of Alzheimer's disease patients," Current Alzheimer Research, vol. 14, no. 7, pp. 760-765, 2017.

[49] L. Racioppi, E. R. Nelson, W. Huang et al., "CaMKK2 in myeloid cells is a key regulator of the immune-suppressive microenvironment in breast cancer," Nature Communications, vol. 10, no. 1, p. 2450, 2019.

[50] X. Wang, G. C. Wang, J. Rong et al., "Identification of steroidogenic components derived from Gardenia jasminoides ellis potentially useful for treating postmenopausal syndrome," Frontiers in Pharmacology, vol. 9, p. 390, 2018.

[51] DIAGRAM, MuTHER, J. S. Kooner et al., "Genome-wide association study in individuals of South Asian ancestry identifies six new type 2 diabetes susceptibility loci," Nature Genetics, vol. 43, no. 10, pp. 984-989, 2011.

[52] A. Goldman, J. L. Smalley, M. Mistry et al., “A computationally inspired in-vivo approach identifies a link between amygdalar transcriptional heterogeneity, socialization and anxiety," Translational Psychiatry, vol. 9, no. 1, p. 336, 2019.

[53] S. R. Oliveira, C. Figueiredo-Pereira, C. B. Duarte, and H. L. A. Vieira, "P2X7 receptors mediate $\mathrm{CO}$-induced alterations in gene expression in cultured cortical astrocytes-transcriptomic study," Molecular Neurobiology, vol. 56, no. 5, pp. 3159-3174, 2019.

[54] D. Wang, S. Zhang, and B. Liu, "TAF5L functions as transcriptional coactivator of MITF involved in the immune response of the clam Meretrix petechialis," Fish \& Shellfish Immunology, vol. 98, pp. 1017-1023, 2020.

[55] D. Seruggia, M. Oti, P. Tripathi et al., "TAF5L and TAF6L maintain self-renewal of embryonic stem cells via the MYC regulatory network," Molecular Cell, vol. 74, no. 6, pp. 11481163.e7, 2019, e7.

[56] H. Ma, H. M. Ng, X. Teh et al., "Zfp322a regulates mouse ES cell pluripotency and enhances reprogramming efficiency," PLoS Genetics, vol. 10, no. 2, article e1004038, 2014. 
[57] H. Fraga, J. J. Bech-Serra, F. Canals, G. Ortega, O. Millet, and S. Ventura, "The Mitochondrial Intermembrane Space Oxireductase Mia40 Funnels the Oxidative Folding Pathway of the Cytochrome $c$ Oxidase Assembly Protein Cox19," The Journal of Biological Chemistry, vol. 289, no. 14, pp. 9852-9864, 2014.

[58] K. A. Lawson, C. M. Sousa, X. Zhang et al., "Functional genomic landscape of cancer-intrinsic evasion of killing by T cells," Nature, vol. 586, no. 7827, pp. 120-126, 2020.

[59] X. Wang, Y. Bai, Y. Han, J. Meng, and H. Liu, " $<$ p $>$ Downregulation of GBAS regulates oral squamous cell carcinoma proliferation and apoptosis via the $\mathrm{p} 53$ signaling pathway $</ \mathrm{p}>$," Oncotargets and Therapy, vol. Volume 12, pp. 3729-3742, 2019.

[60] S. G. Wu, Y. J. Huang, B. Bao et al., "miR-508-5p acts as an anti-oncogene by targeting MESDC1 in hepatocellular carcinoma," Neoplasma, vol. 64, no. 1, pp. 40-47, 2017.

[61] J. Stefani, O. Tschesnokowa, M. Parrilla et al., "Disruption of the microglial ADP receptor P2Y13 enhances adult hippocampal neurogenesis," Frontiers in Cellular Neuroscience, vol. 12, p. 134, 2018.

[62] G. Jin, S. W. Lee, X. Zhang et al., "Skp2-mediated RagA ubiquitination elicits a negative feedback to prevent amino-aciddependent mTORC1 hyperactivation by recruiting GATOR1," Molecular Cell, vol. 58, no. 6, pp. 989-1000, 2015.

[63] L. Gan, A. Seki, K. Shen et al., "The lysosomal GPCR-like protein GPR137B regulates Rag and mTORC1 localization and activity," Nature Cell Biology, vol. 21, no. 5, pp. 614-626, 2019.

[64] J. Kim and E. Kim, "Rag GTPase in amino acid signaling," Amino Acids, vol. 48, no. 4, pp. 915-928, 2016.

[65] J. L. Jewell, Y. C. Kim, R. C. Russell et al., "Differential regulation of mTORC1 by leucine and glutamine," Science, vol. 347, no. 6218, pp. 194-198, 2015.

[66] S. M. Son, S. J. Park, H. Lee et al., "Leucine signals to mTORC1 via its metabolite acetyl-coenzyme A," Cell Metabolism, vol. 29, no. 1, pp. 192-201.e7, 2019, e7.

[67] H. Yang, X. Jiang, B. Li et al., "Mechanisms of mTORC1 activation by RHEB and inhibition by PRAS40," Nature, vol. 552, no. 7685, pp. 368-373, 2017.

[68] P. T. Newton, K. K. Vuppalapati, T. Bouderlique, and A. S. Chagin, "Pharmacological inhibition of lysosomes activates the MTORC1 signaling pathway in chondrocytes in an autophagy-independent manner," Autophagy, vol. 11, no. 9, pp. 1594-1607, 2015.

[69] J. M. Dwyer, J. G. Maldonado-Avilés, A. E. Lepack, R. J. DiLeone, and R. S. Duman, "Ribosomal protein S6 kinase 1 signaling in prefrontal cortex controls depressive behavior," Proceedings of the National Academy of Sciences of the United States of America, vol. 112, no. 19, pp. 6188-6193, 2015.

[70] S. Dutheil, K. T. Ota, E. S. Wohleb, K. Rasmussen, and R. S. Duman, "High-fat diet induced anxiety and anhedonia: impact on brain homeostasis and inflammation," Neuropsychopharmacology, vol. 41, no. 7, pp. 1874-1887, 2016.

[71] S. Jung, S. Choe, H. Woo et al., "Autophagic death of neural stem cells mediates chronic stress-induced decline of adult hippocampal neurogenesis and cognitive deficits," Autophagy, vol. 16, no. 3, pp. 512-530, 2020.

[72] Y. Hasegawa, X. L. Zhu, and A. Kamiya, "NV-5138 as a fastacting antidepressant via direct activation of mTORC1 signaling," The Journal of Clinical Investigation, vol. 129, no. 6, pp. 2207-2209, 2019.
[73] W. Fan, K. Cheng, X. Qin et al., "mTORC1 and mTORC2 play different roles in the functional survival of transplanted adipose-derived stromal cells in hind limb ischemic mice via regulating inflammation in vivo," Stem Cells, vol. 31, no. 1, pp. 203-214, 2013.

[74] B. R. Rosborough, D. Raïch-Regué, B. M. Matta et al., "Murine dendritic cell rapamycin-resistant and rictor-independent mTOR controls IL-10, B7-H1, and regulatory T-cell induction," Blood, vol. 121, no. 18, pp. 3619-3630, 2013. 